\title{
On Generalized Closure Operators in Generalized Topological Spaces
}

\author{
B.K. Tyagi ${ }^{1}$ \\ Department of Mathematics, \\ Atmaram Sanatan Dharma College, \\ University of Delhi, New Delhi 110021, India
}

\author{
Rachna Choudhary ${ }^{2}$ \\ Department of Mathematics, \\ University of Delhi, Delhi 110007, India
}

\begin{abstract}
In this paper, you study (strong) generalized closure operators and their interactions with generalized topologies, (strong) generalized interior operators (ascending, complete) generalized neighbourhood systems and extend the commuting diagram of Cao, Wang and Wang [1] to include strong generalized closure operators.
\end{abstract}

\section{General Terms:}

$54 \mathrm{~A} 05$

\section{Keywords:}

Generalized topological spaces, (strong) Generalized closure and interior operators, (Ascending, Complete) Generalized neighbourhoods systems

\section{INTRODUCTION}

In a series of papers, Császár ([2], [3] and references there in) has laid the foundation of generalized topological spaces which contains the class of topological spaces. These are mathematical structures in which generalized topologies are collections of subsets of a set closed under arbitrary unions. Similarly, the notions of neighbourhood systems and interior operators, are weakened to obtain the notions of generalized neighbourhood system and generalized interior operator. Then their interactions with each other were studied in Császár [2], Shen [5], Min [4], Cao et al. [1]. The notion of complete neighbourhood system was introduced in [5] and it is used to establish that there is a one-to-one correspondence between complete neighbourhood systems and generalized topological spaces. Cao et al. [1], further extended the above result and obtained one-to-one correspondence between the class of generalized topological spaces and the class of strong generalized interior operators and hence also between the class of complete generalized neighbourhood system and the class of

\footnotetext{
${ }^{1}$ Mailing address: Dr. B.K. Tyagi, 620, Modern Apartments, Sector 15, Rohini, Delhi 110085, India

${ }^{2}$ Mailing address: Rachna Choudhary, A-135, Ground floor, Parsvnath Paradise, Mohan Nagar, Ghaziabad 201007, Uttar Pradesh, India
}

strong generalized interior operators. In this paper, we supplement the above work by including in the framework the notion of (strong) generalized closure operators. Then the interaction of generalized closure operator with the generalized topological spaces (complete, ascending) generalized neighbourhood systems and (strong) generalized interior operator is investigated. This brings in analogues of all the relevant results in [5, 4] 1]. Further some new results are required to extend the commuting diagram of Cao et al. [1] to include in it the strong generalized closure operators.

The paper is organized as follows. Section 2 contains preliminaries. Section 3 contains the interaction among generalized topological spaces,(ascending, complete) generalized neighbourhood systems and strong generalized closure operators, which essentially completes the proof that the outer triangles in the diagram in Theorem 4.9 commutes. In Section- 4 , we establish that the other triangles in the above diagram also commute.

\section{PRELIMINARIES}

Let $X$ be a set and $\operatorname{Exp} X$ be the power set of $X$. A subset $\mu$ of $\operatorname{Exp} X$ is called a generalized topology (GT) on $X$ if $\mu$ is closed under arbitrary unions. Note that the empty set $\phi$ is in $\mu$ and the set $X$ may not be in $\mu$. A generalized topological space is a pair $(X, \mu)$, where $\mu$ is a GT on $X$. In a generalized topological space $(X, \mu)$ the elements of $\mu$ are called $\mu$-open sets and their complements are called $\mu$-closed sets. A mapping $\Psi: X \rightarrow$ $\operatorname{Exp}(\operatorname{Exp} X)$ is called a generalized neighbourhood system (GNS) if for each $x \in X$ and the set $A \in \Psi(x), x \in A$. A GNS $\Psi$ is called ascending if for each $x \in X, V \in \Psi(x)$ and $V \subseteq A$ implies that $A \in \psi(x)$.

A GNS $\Psi$ is said to be complete (CGNS) if for each $x \in X$ and $x \in A \in \Psi(x)$ there is a set $O$ such that $x \in O \subseteq A$ and $y \in O$ implies that there exists a set $B \in \Psi(y)$ with $B \subseteq O$.

A mapping $I: \operatorname{Exp} X \rightarrow \operatorname{Exp} X$ is called a generalized interior operator (GIO) if

(i) $I(A) \subseteq A$, and

(ii) $A \subseteq B$ implies $I(A) \subseteq I(B)$ for all $A, B \in \operatorname{Exp}(X)$.

A GIO is said to be strong (SGIO) if $I(I(A))=I(A)$ for all $A \in \operatorname{Exp}(X)$. 
Given one of GT, GNS and GIO on a set $X$, the other two are related via the following definitions $[1,2,4,5]$.

Given $\mu$ :

$$
\begin{aligned}
& \Psi_{\mu}(x)=\{A: x \in M \subseteq A \text { for some } M \in \mu\} . \\
& I_{\mu}(A)=\cup\{M \subseteq A: M \in \mu\} .
\end{aligned}
$$

Given $\mu$ :

$\mu_{\Psi}=\{M \subseteq X: x \in M$ implies that there exists a $V \in \Psi(x)$ such that $V \subseteq M\}$.

$\Psi_{I}(A)=\{x \in M$ : there exists a set $V \in \Psi(x)$ such that $V \subseteq A\}$.

Given $I$ :

$$
\begin{aligned}
& \mu_{I}=\{A \subseteq X: A=I(A)\} . \\
& \Psi_{I}(x)=\{A \subseteq X: x \in I(A)\} .
\end{aligned}
$$

It is obvious that $\Psi_{\mu}$ and $\Psi_{I}$ are GNSs, $I_{\mu}$ and $I_{\Psi}$ are GIOs, and $\mu_{\Psi}$ and $\mu_{I}$ are GTs on $X$. Moreover, $\Psi_{\mu}$ is always complete and $I_{\mu}$ is always strong. It is a shown in Cao et al. [1] that the following diagram commutes:

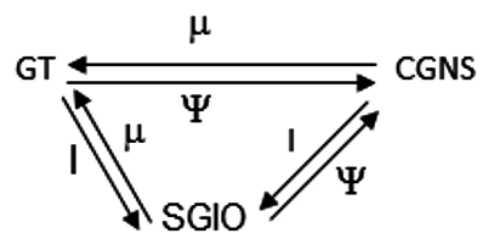

Fig. 1.

TheOREM 2.1 [5, TheOREM 2.4]. . For a GNS $\Psi$ on a set $X$, the following statements are equivalent.

(1) $\psi$ is complete.

(2) $\Psi=\Psi_{\mu_{\psi}}$.

(3) $\Psi=\Psi_{\mu}$ for some $G T \mu$ on $X$.

A mapping $C: \operatorname{Exp}(X) \rightarrow \operatorname{Exp}(X)$ is said to a generalized Closure operator (GCO) if

(i) $A \subseteq C(A)$ and

(ii) $A \subseteq B$ implies $C(A) \subseteq C(B)$ for all $A, B \in \operatorname{Exp}(X)$.

A GCO is said to be strong (SGCO) if $C(C(A))=C(A)$ for all $A$ in $\operatorname{Exp} X$.

It may be noted that if we define the relation $C(A)=X-I(X-A)$, then $I$ is a GIO if and only if $C$ is $\mathrm{GCO}$, and $I$ is SGIO and if and only if $C$ is SGCO. In this sense $C$ and $I$ are considered to be dual operations.

\section{ON GT, GNS, GCO}

Given a GT $\mu$, we define

$$
C_{\mu}(A)=\cap\left\{F: A \subseteq F \text { and } F^{\prime}=X-F \in \mu\right\} .
$$

Given a GNS $\Psi$, we define

$$
C_{\Psi}(A)=\cap\{x: V \in \Psi(x) \text { implies } V \cap A \neq \emptyset\} .
$$

Given a $\operatorname{GCO} C$, we define

$$
\begin{aligned}
& \mu_{C}=\{A: C(X-A)=X-A\} . \\
& \Psi_{C}(x)=\{A: x \in X-C(X-A)\} . \\
& I_{C}(A)=X-C(X-A) .
\end{aligned}
$$

Given GIO $I$, we define

$$
I_{C}(A)=X-I(X-A) .
$$

It is obvious that $C_{\mu}$ is always a SGCO.

THEOREM 3.1. For each GT $\mu$ on a set $X$. The following statements hold.

(1) $\mu=\mu_{C_{\mu}}$.

(2) $\mu=\mu_{\Psi_{\mu}}$.

(3) $C_{\mu}=C_{\Psi_{\mu}}$.

(4) $\Psi_{\mu}=\Psi_{C_{\mu}}$.

(5) $\mu=\mu_{C_{\Psi \mu}}$.

(6) $\mu=\mu_{\Psi_{C_{\mu}}}$.

Proof. (1). Let $A \in \mu$. Since $X-A$ is $\mu$-closed, $C_{\mu}(X-A)=$ $X-A$. Therefore, $A \in \mu_{C_{\mu}}$. Conversely $A \in \mu_{C_{\mu}}$. Then $C_{\mu}(X-A)=X-A$. This means that $A$ is $\mu$-open.

(2). See [1, Theorem 2.1].

(3). $x \in C_{\mu}(A)$ if and only if $x$ is in every $\mu$-closed set containing $A$ if and only if $x$ is not in any $\mu$-open set disjoint from $A$ if and only if every $\mu$-open set containing $x$ intersects $A$ if and only if every $V \in \Psi_{\mu}(x)$ intersects $A$ if and only if $x \in C_{\Psi_{\mu}}(A)$.

(4). $A \in \Psi_{C_{\mu}}(A)$ if and only if $x \in X-C_{\mu}(X-A)$ if and only if $x \notin C_{\mu}(X-A)$ if and only if there exists a $\mu$-closed set $F$ containing $X-A$ such that $x \notin F$ if and only if there exists a $\mu$-open set $M$ such that $x \in M \subseteq A$ if and only if $A \in \Psi_{\mu}(x)$.

(5). (1) and (3) imply (5).

(6). (4) and (2) imply (6).

LEMMA 3.2. For GCO $C$ on a set $X$ we have

(1) $C=C_{\Psi_{C}}$.

(2) $\mu_{C}=\mu_{\Psi_{C}}$.

Proof. (1). We show that $C(A)=C_{\Psi_{C}}(A)$ for each subset $A$ of $X$. Let $x \in C_{\Psi_{C}}(A)$. Then for each $V \in \Psi_{C}(x), V \cap A$ is non-empty. Since $(X-A) \cap A=\phi, X-A$ is not in $\Psi_{C}(x)$. That is, $x$ is not in $X-C(A)$. So that, $x$ is in $C(A)$. Conversely, let $x$ is in $C(A)$. Let $V \in \Psi_{C}(x)$. Then $x$ is not in $C(X-V)$. So $X-V \neq A$. Thus, $V \cap A \neq \phi$.

(2). $A \in \mu_{C}$ if and only if $C(X-A)=X-A$ if and only if $A=X-C(X-A)$ if and only if $A \in \Psi_{C}(x)$ for all $x$ in $A$ if and only if $A \in \mu_{\Psi_{C}}$.

EXAMPLE 3.3. However, the equality $C=C_{\mu_{C}}$ may not hold. We take Example 2.3 in Cao et al. [1] and take $C$ as the dual of the generalized interior operator I defined there, that is $C(A)=X-A(X-A)$. So that $C(\phi)=\phi, C(\{a\})=\{a, c\}$, $C(\{b\})=\{a, b\}, C(\{c\})=\{b, c\}, C(\{b, c\})=C(\{b, c\})=$ $C(\{a, c\})=C(\{a, b\})=C(\{a, b, c\})=X$. So that $\mu_{C}=$ $\{\phi, X\}$. Thus, $C(\{a\})=\{a, c\}$ and $C_{\mu_{C}}(\{a\})=X$.

For a GCO C there may not be any GT $\mu$ such that GCO generated by $\mu$ is $C$. For instance, if in the above example $\mu$ is a GT such 
that $C_{\mu}=C$ then $\mu_{C}=\mu_{C_{\mu}}$ and $C_{\mu_{C}}=C_{\mu_{C \mu}}=C_{\mu}$ by Theorem 3.1(1). Thus, $C_{\mu_{C}}=C$, a contradiction. So it is natural to study the additional restriction necessary on a GCO so that it can be generated by a GT.

LEMMA 3.4. For a GNS $\Psi$ on a set $X, \mu_{\Psi}=\mu_{C_{\Psi}}$.

Proof. Let $A \in \mu_{\Psi}$ and $x$ be a point in $A$. Then there exists, a $V \in \Psi(x)$ such that $V \subseteq A$. So $V \cap(X-A)=\phi$. This means that $x \notin C_{\Psi}(X-A)$. Therefore, $C_{\Psi}(X-A)=X-A$. Thus, $A \in \mu_{C_{\Psi}}$. Conversely, let $A \in \mu_{C_{\Psi}}$ and $x \in A$. Then $A=X-C C_{\Psi}(X-A)$. Therefore, $x \notin C_{\Psi}(X-A)$. So there exist a $V$ in $\Psi(x)$ such that $V \cap(X-A)=\phi$. Therefore, $V \subseteq A$. Thus $A \in \mu_{\Psi}$.

THEOREM 3.5. If $\Psi$ is ascending GNS then $\Psi=\Psi_{C_{\Psi}}$.

Proof. If $A \in \Psi_{C_{\Psi}}(x)$ then $x$ is not in $C_{\Psi}(X-A)$. So there exist a $V \in \Psi(x)$ such that $V \cap(X-A)=\phi$. That is, $V \subseteq A$. Since $\Psi$ is ascending, $A \in \Psi(x)$. Conversely, let $A$ be in $\Psi(\bar{x})$. Since $A \cap(X-A)=\phi, x$ is not in $C_{\Psi}(X-A)$. Thus, $x \in X-C_{\Psi}(X-A)$. Consequently, $A \in \Psi_{C_{\Psi}}(x)$.

THEOREM 3.6. For an ascending GNS $\Psi$ on a set $X$, the following statements are equivalent.

(1) $\Psi$ is complete.

(2) $C_{\Psi}$ is strong.

(3) $C_{\Psi}=C_{\mu}$ for some $G T \mu$ on $X$.

(4) $C_{\Psi}=C_{\mu_{\Psi}}$.

(5) $C_{\Psi}=C_{\mu_{C_{\Psi}}}$.

(6) $\Psi=\Psi_{\mu_{\Psi}}$.

(7) $\Psi=\Psi_{\mu_{\Psi \Psi}}$.

(8) $\Psi=\Psi_{\mu_{C_{\Psi}}}$

Proof. (4) $\Rightarrow(3),(3) \Rightarrow(2)$ and $(4) \Leftrightarrow(5)$ are obvious. So we prove $(2) \Rightarrow(1) \Rightarrow(4)$.

(2) $\Rightarrow$ (1) follows from the fact that $C_{\Psi}$ and $I_{\Psi}$ are dual of each other and the fact $(2) \Rightarrow(1)$ in [2] Theorem 3.2].

$(1) \Rightarrow(4)$. Let $F$ be a $\mu_{\Psi}$-closed set containing $A$. Let $x \in X-F$. Then there exists a set $V \in \Psi(x)$ such that $V \subseteq X-F$. So $V \cap A=\phi$. So that $x$ is not in $C_{\Psi}(A)$. Therefore, $C_{\Psi}(A) \subseteq F$. So $C_{\Psi}(A) \subseteq C_{\mu_{\Psi}}(A)$. Note that in this part we have not used the completeness of $\Psi$. Conversely, let $V \in \Psi(x)$ be such that $V \cap A=\phi$. Since $\Psi$ is complete there exist a set $O$ such that $x \in O \subseteq V$ and $y \in O$ implies that there is a $B \in \Psi(y)$ with $B \subseteq O$. Then $O \in \mu_{\Psi}$ and $O \cap A=\phi$. Therefore $X-O$ is a $\mu_{\Psi}$-closed set containing $A$ and $x$ is not in $X-O$. Thus, $x \notin C_{\mu_{\Psi}}$. This proves that $C_{\mu_{\Psi}}(A) \subseteq C_{\Psi}(A)$. This proves that the first five statements are equivalent.

$(6) \Leftrightarrow(8)$ follows from Lemma 3.4.

$(1) \Leftrightarrow(6)$ comes from Theorem 2.1.

(4) $\Rightarrow$ (7) comes from Theorem 3.5 .

(7) $\Rightarrow(1)$. Since $\mu_{\Psi}$ is a GT, $C_{\mu_{\Psi}}$ is strong. Therefore $\Psi_{C_{\mu_{\Psi}}}=\Psi$ is complete by the implication (2) $\Rightarrow(1)$.

THEOREM 3.7. For a GCO $C$ on a set $X$, the following statements are equivalent.

(1) $C$ is strong.
(2) $\psi_{C}$ is complete.

(3) $\psi_{C}=\psi_{\mu}$ for some GT $\mu$ on $X$.

(4) $\psi_{C}=\psi_{\mu_{C}}$.

(5) $\psi_{C}=\psi_{\psi_{\mu_{C}}}$.

(6) $C=C_{\mu_{C}}$.

(7) $C=C_{\mu_{\psi_{C}}}$.

(8) $C=C_{\psi_{\mu_{C}}}$.

PRoOF. (1) $\Rightarrow(2) . \Psi_{C}$ is complete if and only if $C_{\psi_{C}}$ is strong by Theorem 3.6 if and only if $C$ is strong by Lemma 3.2.

(1) $\Rightarrow(4) \mu_{C}=\mu_{\psi_{C}}$ by Lemma 3.2. Since $C$ is strong, $\Psi_{C}$ is complete. So by Theorem 2.1 $\Psi_{C}=\Psi_{\mu_{\psi_{C}}}=\psi_{\mu_{C}}$.

(4) $\Rightarrow(3)$. Obvious.

(4) $\Rightarrow$ (2) follows from definition of $\Psi_{\mu}$.

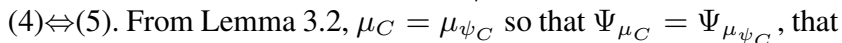
is, $\Psi_{C}=\Psi_{\mu_{\psi_{C}}}$ by (4). Similarly, $\Psi_{C}=\Psi_{\mu_{\psi_{C}}}=\Psi_{\mu_{C}}$ using Lemma 3.2.

(1) $\Rightarrow(6)$. If $C$ is strong then $\mathrm{CA}$ is a $\mu_{C}$-closed set containing $A$. Consequently, $C_{\mu_{C}}(A) \subseteq C A$. On the other hand, if $A \subseteq F$ and $F$ is $\mu_{C}$-closed, then $C A \subseteq C F=F$. So $C A \subseteq C_{\mu_{C}}(A)$.

(6) $\Rightarrow(1)$. We prove $C C A \subseteq C A$. Suppose that $x$ is not in $C A$ and $C A=C_{\mu_{C}}(A)$. So there exists a $\mu_{C}$-closed set $F$ such that $A \subseteq F$ and $x$ is not in $F$. Now $C C A \subseteq C C F=C F=F$. Therefore, $x$ is not in $C C A$. Thus $C$ is strong.

(6) $\Leftrightarrow$ (7) follows from Lemma 3.2(2).

(4) $\Rightarrow(8)$ follows from Lemma 3.2(1).

$(8) \Rightarrow(1)$. Since $\mu_{C}$ is a GT, $\Psi_{\mu_{C}}$ is complete. So $C_{\psi_{\mu_{C}}}=C$ is strong by Theorem 3.6.

\section{ON GT, GNS, GIO AND GCO}

TheOrem 4.1. For a GCO $C$ on a set $X$ the following statements hold

(1) $\psi_{C}=\psi_{I_{C}}$.

(2) $I_{C}=I_{\psi_{C}}$.

(3) $C=C_{I_{C}}$.

Proof. (1). Let $A \in \psi_{I_{C}}(x)$. Then $x \in I_{C}(A)=X-C(X-A)$. Thus, $A \in \Psi_{C}(x)$. Conversely, if $A \in \Psi_{C}(x)$ then $x \in X-C(X-A)$. Therefore, $x \in I_{C}(A)$. So $A \in \Psi_{I_{C}}(x)$.

(2). Let $A$ be a subset of $X$ and $x \in I_{\psi_{C}}(A)$. Then there exists a set $V \in \psi_{C}(x)$ such that $V \subseteq A$. Then $x \in X-C(X-V)$ and $V \subseteq A$. Therefore, $x \in X-C(X-A)=I_{C}(A)$. Conversely, let $x \in I_{C}(A)=X-C(X-A)$ then $A \in \Psi_{C}(x)$. Therefore, $x \in I_{\psi_{C}}(A)$.

(3). For a subset $A$ of $X, C_{I_{C}}(A)=X-I_{C}(X-A)=$ $X-(X-C A)$.

THEOREM 4.2. For a GNS $\Psi$ on a set $X$, the following statement holds.

(1) $I_{\Psi}=I_{C_{\Psi}}$.

(2) $C_{\Psi}=C_{I_{\Psi}}$.

Proof. (1). Let $A$ be a subset of $X$ and $x \in I_{C_{\Psi}}(A)=$ $X-C_{\Psi}(X-A)$. Then there exists a set $V \in \Psi(x)$ such that $V \cap(X-A)=\phi$. Thus, $V \subseteq A$. Therefore, $A \in \Psi(x)$. 
Conversely, let $A \in \psi(x)$. Then $x$ is not in $C_{\Psi}(X-A)$. Therefore, $x \in I_{C_{\Psi}}(A)$.

(2). Let $A$ be a subset of $X$ and $x \in C_{I_{\psi}}(A)$. Then for all $V \in \Psi(x), V \cap A \neq \phi$. Thus, $x \in C_{\Psi}(A)$. Conversely, let $x \in C_{\Psi}(A)$. Then every $V$ in $\Psi(x)$ intersects with $A$. Therefore, $x$ is not in $I_{\Psi}(X-A)$. Thus, $x \in C_{I_{\Psi}}(A)$.

THEOREM 4.3. For a GIO $I$ on a set $X$, the following statements holds.
(1) $\Psi_{I}=\Psi_{C_{I}}$.
(2) $C_{I}=C_{\Psi_{I}}$.
(3) $I_{C_{I}}=I$.

Proof. (1). Let $A \in \Psi_{C_{1}}(x)$. Then $x \in X-C_{I}(X-A)=$ $I(A)$. Therefore $A \in \Psi_{I}(A)$. Converse is obvious.

(2). Let $x$ be a point not in $C_{\Psi_{I}}(A)$. Then there exists a set $V$ in $\Psi_{I}(x)$ such that $V \cap A=\phi$. Then $x \in I(V)$ and $V \subseteq X-A$. Then $x \in I(X-A)$. Therefore, $x$ is not in $C_{I}(A)$. Conversely, if $x$ is a point not in $C_{I}(A)$ then $x \in I(X-A)$. Therefore, $X-A \in \Psi_{I}(x)$ and $(X-A) \cap A=\phi$. Consequently, $x$ is not in $C_{\Psi_{I}}(A)$.

(3). Obvious.

THEOREM 4.4. For a GT $\mu$ on $X$, the following statements hold

(1) $\mu=\mu_{I_{C \mu}}$.

(2) $\mu=\mu_{C_{I \mu}}$.

Proof. (1). $A \in \mu_{I_{C_{\mu}}}$ if and only if $A=I_{C_{\mu}}(A)=$ $X-C_{\mu}(X-A)$ if and only if $C_{\mu}(X-A)=X-A$ if and only if $A \in \mu$.

(2). $A \in \mu_{C_{I_{\mu}}}$ if and only if $X-A=C_{I_{\mu}}(X-A)=X-I_{\mu}(A)$ if and only if $I_{\mu}(A)=A$ if and only if $A \in \mu$. hold.

THEOREM 4.5. For a SGIO I on $X$, the following statements

(1) $I=I_{\mu_{C_{I}}}$.

(2) $I=I_{C_{\mu_{I}}}$.

Proof. (1). Let $A \subseteq X$ and $x \in I_{\mu_{C_{I}}}(A)$. Then there exists a set $M \in \mu_{C_{I}}$ such that $x \in M \subseteq A$. Then $C_{I}(X-M)=X-M$. Therefore, $X-I M=X-M$. Thus, $I M=M$ and $x \in M \subseteq A$. Therefore, $x \in I(A)$. Conversely, let $x \in I(A)$. Since $I$ is strong $X-I(I(A))=X-I(A)$ and $x \in I(A) \subseteq A$. Thus, $C_{I}(X-(I(A)))=X-I(I(A))=X-I(A)$. Therefore $I(A) \in \mu_{C}$. Thus, $x \in I_{C_{\mu_{I}}}(A)$.

(2). Let $x \in I_{C_{\mu_{I}}}(A)-X-C_{\mu_{I}}(X-A)$. This means that there exists a set $F$ such that $X-A \subseteq F, F^{\prime}=X-F \in \mu_{I}$ and $x$ is not in $F$. Therefore, $x \in F^{\prime} \in \mu_{I}$. Thus, $I\left(F^{\prime}\right)=F^{\prime}$ and $x \in F^{\prime} \subseteq A$. So $x$ is in $I(x)$. Conversely, let $x \in I(A)$. Since $I$ strong, $I(A) \in \mu_{I}$. Since $X-A \subseteq X-I(A)$ it follows that $x$ is not in $C_{\mu_{I}}(X-A)$. Therefore, $x$ is in $I_{C_{\mu_{I}}}(A)$. hold.

(1) $C=C_{I_{\mu_{C}}}$.
(2) $C=C_{\mu_{I_{C}}}$.
(3) $C=C_{\Psi_{I_{C}}}$.
(4) $C=C_{I_{\Psi_{C}}}$.

Proof. (1). For a subset $A$ of $X$, let $x \in C_{I_{\mu_{C}}}(A)=$ $X-I_{\mu_{C}}(X-A)=X-\cup\left\{M \subseteq X-A: M \in \mu_{C}\right\}=$ $X-\cup\{M \subseteq X-A: C(X-M)=X-M\}$. Then for $M \subseteq X-A$ such that $C(X-M)=X-M, x \notin M$. Let us take $M=X-C(A)$. Then $M \subseteq X-A$ and $C(X-M)=C(C(A))=C(A)=X-M$. So that $x$ is not in $X-C A$. Conversely, let $x$ be in $C A$. Let $M \subseteq X-A$ be such that $C(X-M)=X-M$. Then $A \subseteq X-M$ so that $C A \subseteq X-M$. Thus, $M \subseteq X-C A$. Therefore, $x$ is not in $\cup\{M \subseteq X-A: C(X-M)=X-M\}=I_{\mu_{C}}(X-A)$. It means that $x \in C_{I_{\mu_{C}}}(A)$.

(2). For a subset $A$ of $X$, we have $C_{\mu_{I_{C}}}(A)=\cap\{F: A \subseteq F$ and $\left.X-F \in \mu_{I_{C}}\right\}=\cap\left\{F: A \subseteq F\right.$ and $I_{C}(X-F)=X-F=$ $\cap F: A \subseteq F$ and $X-C F=X-F=\cap\{F: A \subseteq F$ and $C F=F\} \subseteq C(A)$ since $C$ is strong, $A \subseteq C(A)=C(C(A))$. Conversely, if $A \subseteq F$ and $C(F)=F$ then $C(A) \subseteq(F)=F$. Consequently, $C(A) \subseteq C_{\mu_{I_{C}}}(A)$.

(3) follows from Lemma 3.2 and Theorem 4.1.

(4) follows from Theorem 4.1(2) and (3).

THEOREM 4.7. If $\Psi$ is ascending GNS on $X$. Then the following statements hold.

(1) $\Psi=\Psi_{I_{C_{\Psi}}}$.

(2) $\Psi=\Psi_{C_{I_{\Psi}}}$.

Proof. (1) $\Psi=\Psi_{I_{\Psi}}$ by [2, Theorem 3.5]. Now (1) follows from Theorem 4.2.

(2). $\Psi=\Psi_{C_{\Psi}}$ by Theorem 3.5(2) now follows from Theorem 4.2. hold.

THEOREM 4.8. For GIO I on a set $X$, the following statements

(1) $I=I_{\Psi_{C_{I}}}$.

(2) $I=I_{C_{\Psi_{I}}}$.

Proof. (1). Follows from [5], Theorem 3.5] and Theorem 4.3. (2). Follows from Theorem 4.3(2) and (3).

The following theorem extends the diagram mentioned earlier.

THEOREM 4.9. The following diagram commutes, where the objects are the classes of GT's, CGNSs, SGIOs and SGCOs.

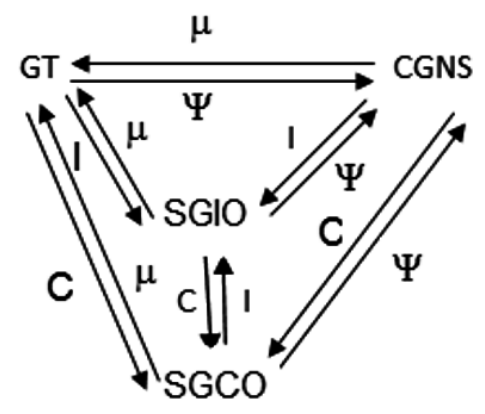

Fig. 2. 


\section{CONCLUSION}

The notion of strong generalized closure operator studied in this paper constitute a fundamental tool in the study of generalized topological spaces.

\section{REFERENCES}

[1] B. Wang C. Cao and W. Wang. Generalized topologies, generalized neighbourhood systems and generalized interior operators. Acta Math. Hungar, 132(4):310-315, oct 2011.

[2] A. Császár. Generalized topology, generalized continuity. Acta Math. Hunger, 19:351-357, 2002.

[3] A. Császár. On generalized neighbourhood systems. Acta Math. Hunger, 2:395-400, 2008.

[4] W.K. Min. Some results on generalized topological spaces and generalized systems. Acta Math. Hungar., 108(1-2):171-181, 2008.

[5] R. Shen. Complete generalized neighbourhood system. Acta Math. Hungar, 129(1-2):160-165, 2010. 